\title{
Recent Advances in the Application of Differential Equations in Mechanical Engineering Problems
}

\author{
Rahmat Ellahi $\left(\mathbb{D},{ }^{1,2}\right.$ Constantin Fetecau, ${ }^{3}$ and Mohsen Sheikholeslami ${ }^{4}$ \\ ${ }^{1}$ Department of Mathematics \& Statistics, IIUI, Islamabad, Pakistan \\ ${ }^{2}$ University of California, Riverside, CA, USA \\ ${ }^{3}$ Academy of Romanian Scientists, 050094 Bucuresti, Romania \\ ${ }^{4}$ Department of Mechanical Engineering, Babol Noshirvani University of Technology, Babol, Iran
}

Correspondence should be addressed to Rahmat Ellahi; rahmatellahi@yahoo.com

Received 9 January 2018; Accepted 9 January 2018; Published 5 March 2018

Copyright (C) 2018 Rahmat Ellahi et al. This is an open access article distributed under the Creative Commons Attribution License, which permits unrestricted use, distribution, and reproduction in any medium, provided the original work is properly cited.

Differential equations arising in mechanics, physics, engineering, biological sciences, economics, and other fields of sciences are classified as either linear or nonlinear and formulated as initial and/or boundary value problems. For nonlinear problems, it is mostly difficult to obtain closedform solutions. However, recent advances made by the guest editors in the application of differential equations in the simulation and modeling of fluids, and particularly in the rheological characteristics of fluids, have motivated us to consider this special issue. Such models are described by several constitutive relations as opposed to one, and as a result they have found wide range of applications in many areas of engineering and sciences, such as mechanical engineering, for example, in solid mechanics; aerospace engineering, for example, in aerodynamics; geophysical/astrophysical phenomena, for example, in weather-forecasting and spaceexploration; and biomedical and health sciences, for example, in infectious disease control.

At present, there exist several theoretical and experimental problems in engineering which are still unsolved. The aim of the special issue was to present recent advances at attempts to extend exact, semianalytic, and computational methods for mathematical models in science and engineering, in both the theoretical and applied aspects. In addition, it was also hoped that it will serve as a forum for presenting new and novel developments in the application of differential equations in several branches of science and engineering. Due to vast range of application the investigations on this special issue were still scant. In order to fill this gap, researchers were invited to contribute original research and review articles.
We received a total of 35 submissions for possible publication. We tried to focus on material that either advances the state-of-the-art of experimental, numerical, and theoretical methodologies or extends the bounds of existing methodologies to new contributions in mechanical sciences. After comprehensive peer review only 11 out of 35 submitted papers have been accepted for final publication where the authors are from geographically distributed countries (USA, China, UK, Japan, South Africa, Egypt, Romania, Iran, Pakistan, Cameroon, and Saudi Arabia). This reflects the high impact of the proposed topic, the academic standings of guest editors, and well organization of journal's Editorial Board of this special issue.

In the paper "Mathematical Model for Electric Field Sensor Based on Whispering Gallery Modes Using Navier's Equation for Linear Elasticity," A. R. Ali and M. A. Kamel presented a mathematical model of an electric field sensor based on the whispering gallery mode (WGM). A microsphere is used to measure the applied electric field due to the electrostriction effect. Generally, this electric field induces body or surface forces which deform the sphere changing its size and causing shifts in its WGM transmission spectrum. The applied electric field can be obtained by calculating these shifts. For some dielectric materials the volumetric body force is zero and the deformation due to the pressure forces is obtained using Navier's equation for the linear elasticity at steady state. The sensor characteristics and behavior have been verified by numerical finite element studies.

In the paper "Applications of Group Theoretical Methods to Non-Newtonian Fluid Flow Models: Survey of Results," 
T. Aziz and F. M. Mahomed offered a comprehensive survey of the studies which deal with flow models of non-Newtonian fluids using the Lie group method and conditional symmetries. This method is used to solve differential equations having a sufficient number of symmetries and its application does not depend of the type of equation or the number of variables. Moreover, it can be applied to any class of differential equations. Recently, the Lie symmetry analysis has been widely applied in different areas of mathematics, mechanics, physics, and applied sciences. It became an efficient tool for solving nonlinear problems which are formulated in terms of ordinary or partial differential equations. In order to prove this, the authors illustrated the use of this method on wellknown Korteweg-de Vries equation and different motion problems of power-law, Sisko, Jeffrey, Williamson, second grade, modified second grade, power law of second grade, Maxwell, micropolar, Eyring-Powell, Oldroyd-B, third-grade, fourth-grade, couple stress, Phan-Tien-Taner, and Casson fluids or nanofluids. A brief version of the nonclassical symmetry method for partial differential equations is also included and the present survey provides a platform for researchers to apply this method to tackle nonlinear problems of Fluid Mechanics.

In the paper "A Self-Adaptive Numerical Method to Solve Convection-Dominated Diffusion Problems," Z.-W. Cao et al. reported new numerical approach to solve convectiondominated diffusion problems. Common adaptive mesh approaches involve complex adaptive operations but this method was developed as an adaptive mesh method which is free from complex adaptive operations. To solve the timedependent problem, movement of mesh points is tracked according to the governing equation, while their values are fixed. Adaptivity of the mesh points is automatically achieved during the course of solving the discretized equation. Several verifications have been applied. All of them indicate good agreement.

In the paper "Finite Element Model for Linear Elastic Thick Shells Using Gradient Recovery Method," A. G. Feumo et al. investigated linear elastic thick shells. They employed Finite Element Model (FEM). Simulation on increasing values of the ratio of the shell shows impact of the $\mathrm{N}$ $\mathrm{T}$ model especially on transverse stresses because of the significant energy contribution due to the third fundamental form tensor present in the kinematics of this model. The analysis of the thickness ratio shows difference between the classical K-L theory and N-T model when the ratio is greater than 0.099 .

In the paper "Unsteady Bioconvection Squeezing Flow in a Horizontal Channel with Chemical Reaction and Magnetic Field Effects," Q. Zhao et al. reported the transient squeezing flow in a channel in existence of magnetic field and chemical reaction. The fully coupled nonlinear systems describing the total mass, momentum, thermal energy, mass diffusion, and microorganisms equations are reduced to a set of ordinary differential equations via a set of new similarity transformations. The detailed analysis illustrating the influences of various physical parameters such as the magnetic, squeezing, and chemical reaction parameters and the Schmidt and Prandtl numbers on the distributions of temperature and microorganisms as well as the skin friction and the Nusselt number was presented. They found that flow field, temperature, and chemical reaction profiles are significantly influenced by magnetic parameter, heat generation/absorption parameter, and chemical parameter.

In the paper "Level-of-Service Based Hierarchical Feedback Control Method of Network-Wide Pedestrian Flow," Z. Zhang et al. introduced a network-wide pedestrian flow model based on the modified cell transmission model which describes the link flow as ordinary differential equations. Level-of-Service Based Hierarchical Feedback Control Method was applied to control the crowd of a hall and the comparison of the simulation results in the controlled and uncontrolled scenarios shows that the proposed HFCM has the capability to suggest the optimal link inflows and walking speeds in real time to meet the LOS requirement.

In the paper "A Comparative Study on Evaluation Methods of Fluid Forces on Cartesian Grids," T. Nonomura and J. Onishi proposed a simple method for evaluating the forces acting on flows around bodies in the immersed boundary scenario. This method has been developed by employing a novel mesh-face integration method and an extrapolation method for evaluating pressure and shear stresses at the mesh faces, such as the first-order, ghostcell, or ghost-fluid methods. The present method is, in principle, advantageous over the conventional methods based on control volumes in that pressure and shear stress can be evaluated separately. Moreover, authors have applied the present method to the computation of the drag force acting on a sphere in Stokes flow and have investigated the effects of grid spacing and extrapolation methods on the errors originating from the present force estimation method by using the existing analytical solutions. In addition, we have addressed the computational costs. As a result, the accuracy of the proposed mesh-based scheme has been proven to be comparable to that of the polygon-based scheme, which is commonly adopted in straightforward implementation. This indicates that the proposed scheme works better than the polygon-based one when complex geometries are involved, since its implementation is simple and its computational cost is low. The error sources in the proposed implementation are sourced from (1) the surface area vector of the staircase body shape and (2) the approximated shear stress. Of these, error in the evaluated shear stress dominates and is significant. If the shear stress is appropriately evaluated, the fluid force can be accurately obtained by summing over the mesh faces, because the surface area vector components converge with increasing grid density while the surface area does not. The shear stress is adequately evaluated by the second-order finite differencing scheme with the ghost-cell or ghost-fluid method. Sometimes, it is difficult to estimate the shear stress accurately with this method by its complex shape. It should be noted that this difficulty is caused by the immersed boundary methods themselves and the present idea using the staircase integration does not have difficulty.

In the paper "An Improved Finite Element Meshing Strategy for Dynamic Optimization Problems," M. Gong et al. have studied a finite element mesh-partitioning strategy based on the direct transcription method to solve the optimal 
control problem with error estimation on noncollocation point. Firstly, the simultaneous strategy based on the finite element is used to transform the differential and algebraic optimization problems (DAOPs) into large scale nonlinear programming problems. Then, the state variables of the reaction process are obtained by simulating with fixed control variables. The noncollocation points are introduced to compute the error estimates of the state variables at noncollocation points. Finally, in order to improve the computational accuracy with less finite element, moving finite element strategy was used for dynamically adjusting the length of finite element appropriately to satisfy the set margin of error. The proposed strategy is applied to two classical control problems and a large scale reverse osmosis seawater desalination process. Computing result shows that the proposed strategy can effectively reduce the computing effort with satisfied accuracy for dynamic optimization problems.

In the paper "CuO-Water Nanofluid Magnetohydrodynamic Natural Convection inside a Sinusoidal Annulus in Presence of Melting Heat Transfer," M. Sheikholeslami et al. examined the impact of nanofluid natural convection due to magnetic field in existence of melting heat transfer by means of CVFEM. The KKL model is taken into account to obtain properties of $\mathrm{CuO}-\mathrm{H} 2 \mathrm{O}$ nanofluid. Roles of melting parameter $(\delta), \mathrm{CuO}-\mathrm{H} 2 \mathrm{O}$ volume fraction $(\phi)$, Hartmann number $(\mathrm{Ha})$, and Rayleigh $(\mathrm{Ra})$ number are depicted in outputs. Results depict that temperature gradient improves with rise of Rayleigh number and melting parameter. Nusselt number detracts with rise of $\mathrm{Ha}$. At the end, a comparison as a limiting case of the considered problem with the existing studies is made and found in good agreement. It is observed that adding magnetic field makes the temperature gradient reduce due to domination of conduction mechanism in high Hartmann number.

In the paper "Global Attractor of Thermoelastic Coupled Beam Equations with Structural Damping," P. Shi et al. have inspected the existence of a global attractor for a class of $n$-dimension thermoelastic coupled beam equations with structural damping within bounded domain of both continuous nonnegative nonlinear real functions. They used classical Galerkin method to establish the existence and uniqueness of regular solution to problem. Obtained results have been validated by several examples.

In the paper "Frequency Equation of Flexural Vibrating Cantilever Beam Considering the Rotary Inertial Moment of an Attached Mass," B. Wang et al. addressed the derivation of the frequency equation of flexural vibrating cantilever beam considering the bending moment generated by an additional mass at the free end of beam, not just the shear force. It is a transcendental equation with two unambiguous physical meaning parameters which can be defined as the ratio of rotary mass moment of inertia and the ratio of the mass, respectively. It is found that as the ratio of rotary mass moment of inertia increases, the natural frequency climbs. Even a little increment of the ratio may cause higher variance between considering and not considering the rotary mass moment of inertia, especially for the high natural frequency. The results show that the inertial moment of the mass has the significant effect on the natural frequency and the shape mode. And it is more reasonable to use this frequency equation to analyze vibration and measure modulus.

\section{Acknowledgments}

The guest editorial team would like to thank all authors for contributing their original work to this special issue. A large number of papers could not be accommodated: while the submission was technically correct, they were inappropriate for the scope of this special issue. The editorial team would also like to thank all anonymous reviewers for their valuable input, efforts, and cooperation during review process. We also acknowledge the entire staff of journal's Editorial Board for providing us with their support regarding this special issue. We hope that the scientists who are working in the same regime not only will enjoy this special issue but would also appreciate the efforts devoted to it by the entire team.

Rahmat Ellahi

Constantin Fetecau

Mohsen Sheikholeslami 


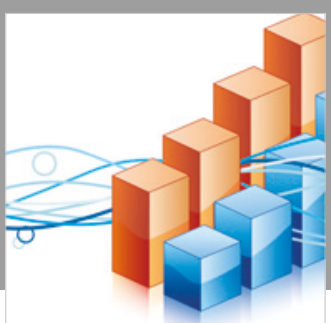

Advances in

Operations Research

\section{-n-m}
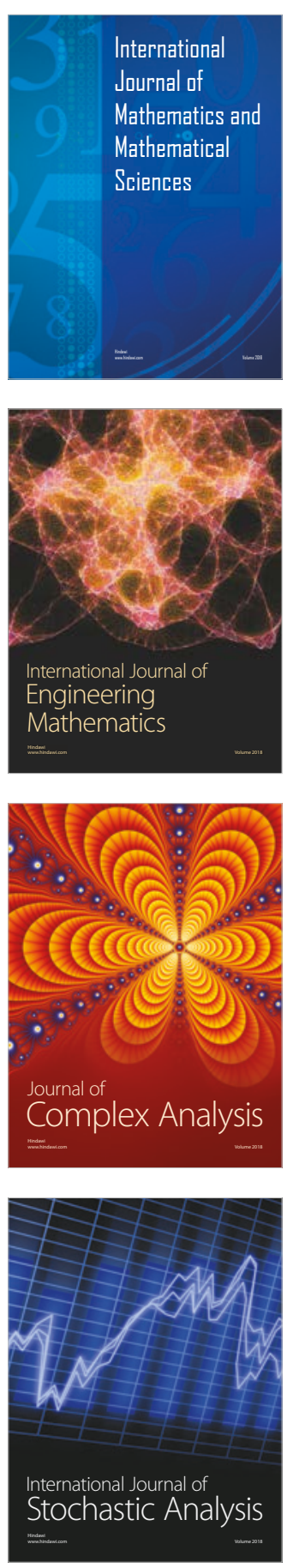
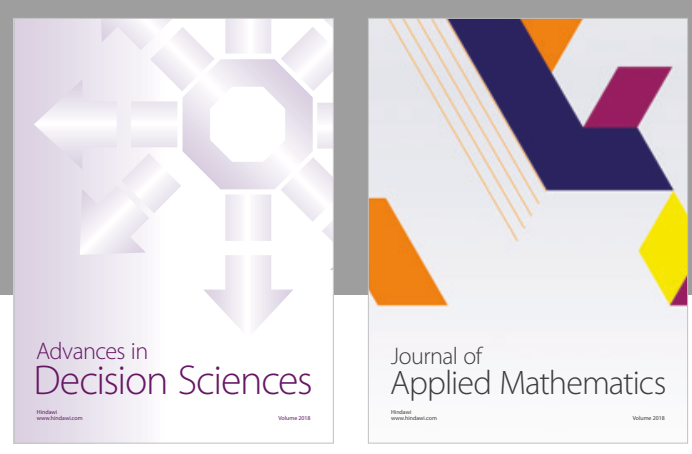

Journal of

Applied Mathematics
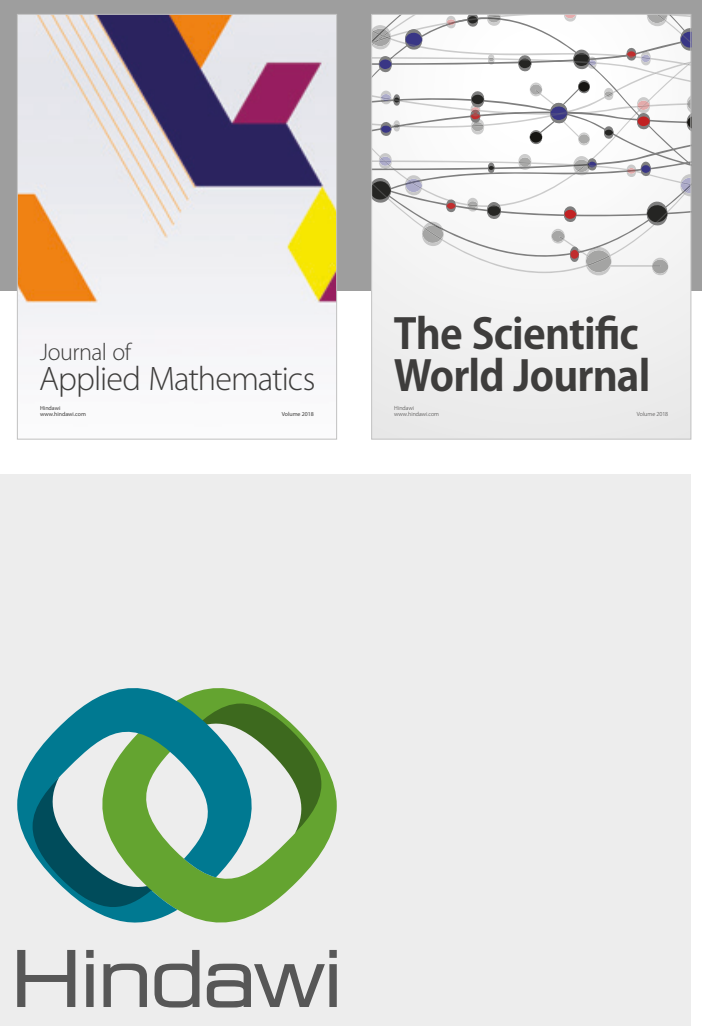

Submit your manuscripts at

www.hindawi.com

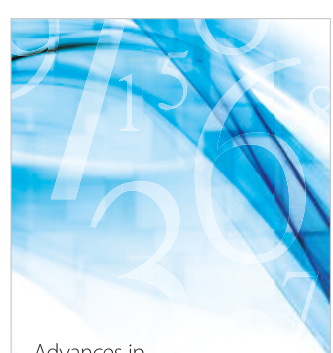

Advances in
Numerical Analysis
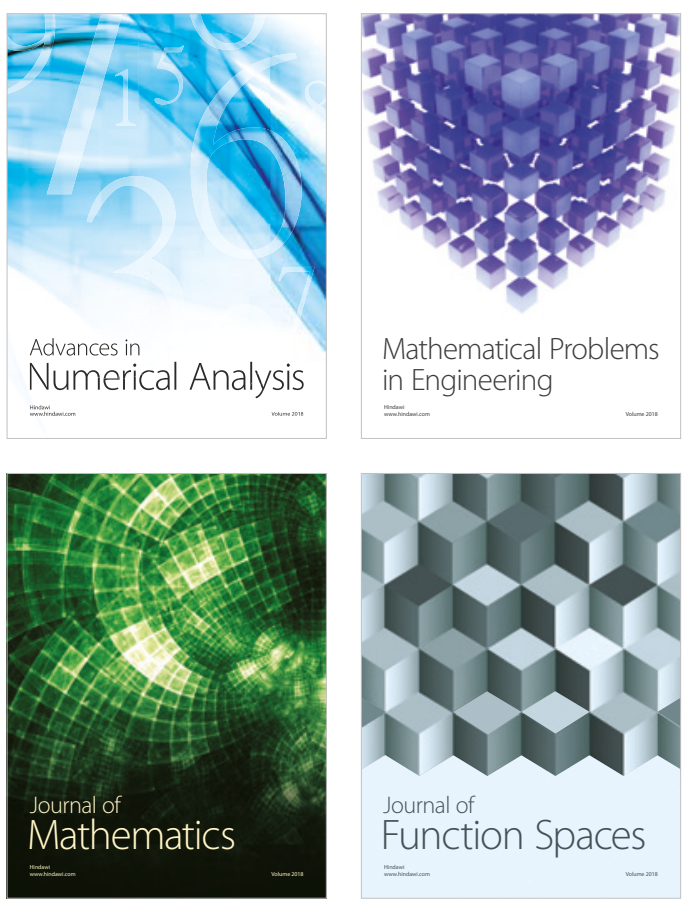

Mathematical Problems in Engineering

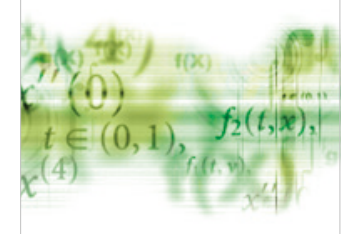

International Journal of

Differential Equations

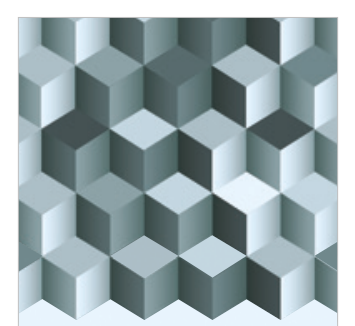

Journal of

Function Spaces

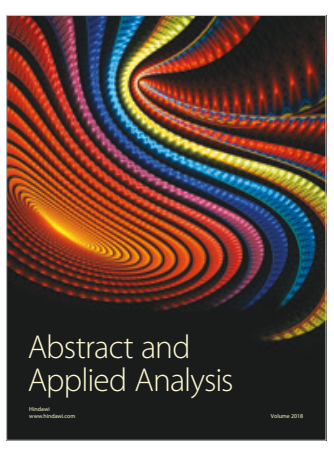

The Scientific

World Journal

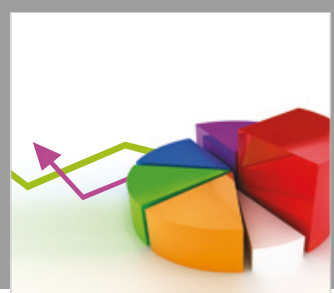

Journal of

Probability and Statistics
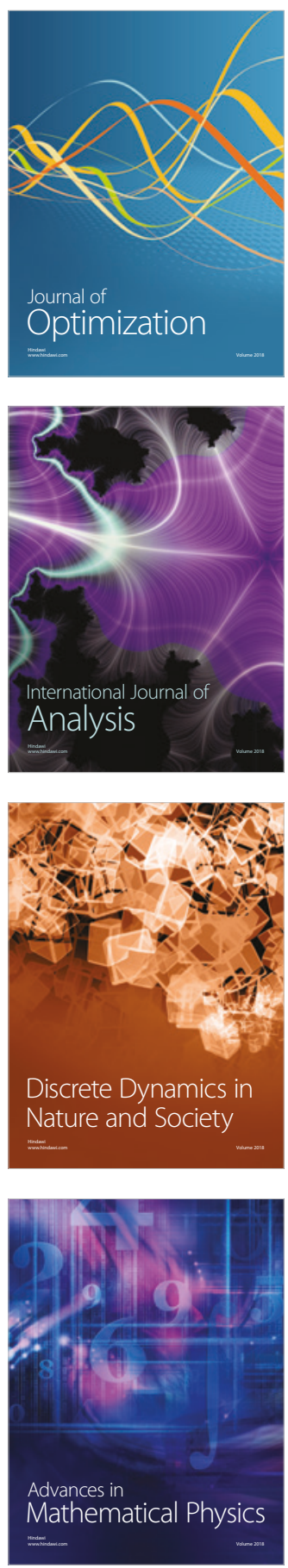